\title{
Efficacy Studies of Hepatoprotective Drug Isolated from Eclipta prostrata. $L$.
}

\author{
Veena ${ }^{1,}$ Jolly Mariam Johny $*^{2}$ \\ ${ }^{I}$ Sundharapuram, Coimbatore, Tamil Nadu, India. \\ ${ }^{* 2}$ Department of Microbiology, Bharathiar University, Coimbatore, Tamil Nadu, India
}

\begin{abstract}
$\overline{\text { Abstract : In the commercial market, medicinal herbs are used as raw drugs, extracts or tinctures. Isolated }}$ active constituents are used for applied research. Medicinal herbs are significant source of hepatoprotective drugs, Eclipta prostrata. L. with active principle Wedelolactone is the prime focus. In the present study to derive hepatoprotective drug, the herb E. prostrata was grown in the Periyar Maniammai College Campus, Vallam and $1 \mathrm{~kg}$ of the plant leaves were collected and shade dried. Dried and powdered maetrial of E. prostrata was extracted with $80 \%$ ethanol. The active principle was isolated and identified by chromatographic technique. The protective effect of E. prostrata on Carbon tetrachloride induced acute liver damage was also studied with male wistar albino rats (180-230g). The protective effect was also undergone for histological studies.
\end{abstract}

Keywords: Eclipta prostrata. L., Wedelolactone, Histological studies, Carbon tetrachloride induced acute liver damage

Accepted Date: 29 June 2013

\section{Introduction}

Plants have always been a common source of medicament either in the form of traditional preparation or pure active principles. Synthetic products of the modern civilization have overshadowed the usage of herbal medicines. But once having realized their serious side effects people are going back to nature with hopes of safety and security. Medicinal herb is a considered to be a chemical factory as it contains multitude of chemical compounds like alkaloids, glycosides, saponins, resins, oleoresins, sesquiterpenes lactones, and essential and fixed oils. World Health Organization has estimated that $80 \%$ of the people in the world rely on traditional medicine for primary healthcare needs? And of the 119 plants based drugs used today by modern medicine about $74 \%$ are from plants traditionally used as herbal cures ${ }^{[1]}$. It is estimated that the market potential for herbal drugs in the western world alone could range from US \$ 4.9 billion to 47 billion by the year 2000. It is been estimated that higher plants produce more than 100,000 secondary metabolites of which only $15-20 \%$ have been chemically characterized ${ }^{[2]}$.

Eclipta prostrata. L. belongs to the family Asteraceae are the most commonly used hepatoprotective herb in the Indian systems of Medicine. The general plant description of E. Prostrata and its medicinal properties was reported by ${ }^{[3]} ;{ }^{[4]} ;{ }^{[5]} ;{ }^{[6]}$. Root of E. prostrata yielded hientricontanol, hepatocosanol-14 and stigmasterol ${ }^{[7]}$. The effect of $E$. prostrata in hepatic injury was studied by ${ }^{[8]}$, and reported that the alcoholic extract of E.prostrata when administered orally in male rats of Hoffkine strain in a dose of $20 \mathrm{mg} / \mathrm{kg}$ body weight once a day for seven days, paracetamol, and afflatoxin induced liver damages. A definite immune inactive property of E. prostrata against, the surface antigen of hepatitis B virus has been reported by ${ }^{[9]}$. Indigenous drugs containing E. prostrata as one of the constituents also showed positive result as antiviral hepatitis in clinical research ${ }^{[10]}$; ${ }^{[11]}$. Beneficial effect of aerial parts of E. prostrata on inflammation and liver injury on rats has been reported by ${ }^{[12]}$. The main anti-hepatotoxic principle Wedelolactone was extracted from the leaves of E. prostrata by ${ }^{[13],[14]}{ }^{[15]}$ Bhargava et al isolated the desmethyl wedelolactone and its glucoside from the leaves of E. prostrata.

\section{Mate rials And Methods}

\subsection{Collection, Phytochemical screening, and Processing of Plant material}

The fresh plant parts of Eclipta prostrata. L. was collected from Periyar Maniammai College of Technology for Women, Vallam, Thanjavur. The habitat, plant species, and the plant parts of E. prostrata were identified, compare, and confirmed with the species preserved in the herbarium of Tamil University, Thanjavur. The leaves were allowed to shade dried for three days, pulverized, and used for photochemical analysis and extraction of the active constituent. 


\subsection{Chromatographic and histological studies of he patoprotective drug Wedelolactone}

Wedelolactone was detected by the procedure laid down by Das et al ${ }^{(16) .}$ Ethanolic extract of the plant material was prepared, filtered, and concentrated to $1 \mathrm{ml}$. 20 microl of the extract was spotted on TLC plates. The samples were spotted on activated plates and RF values were calculated. HPLC analysis was performed with a Waters Associates instrument using reverse phase C-18 columns of $14 \times 0.8 \mathrm{~cm}$ and water-methanol (90:10v/v) as developing solvent, and absorbance was detected using UV absorption at $254 \mathrm{~nm}$.

4-6 weeks male Wister albino rats 4-6 weeks, 180-230g were divided into five groups with six animals each.

Group 1 - Rats were given normal saline $=10 \mathrm{ml} / \mathrm{Kg} /$ day

Group 2 - Rats were given saline (Control)

Group 3 - Rats were given standard

Group 4 and 5 was administered with $100 \mathrm{mg} / \mathrm{Kg}$ and $200 \mathrm{mg} / \mathrm{Kg}$ E. prostrata extracts in PEG.

After 24 hours the rats were sacrificed and blood was collected in a non heparinisede tube. Seru m was separated by centrifugation at $2500 \mathrm{rpm}$ for $10 \mathrm{~min}$ ad used for the determination of SGOPT, SGOT, ALT, CP, and bilirubin levels. Invitro determination of SGOT was done by the method of Ladue et al (1954). For histological evaluation, hepatative tissues sections were cut and stained with haemto xy lin-eosin for histological examination.

\section{Results And Discussion}

The ethanol soluble plant constituents are extracted and concentrated by distillation process and subjected for further phytochemical analysis. Qualitative phytochemical analysis reveals the presence of carbohydrates, alkaloids, phytosterols, saponins, tannins and phenolic compounds, flavanoids, and lignin in the leaf extract of E. prostrata.

The presences of the phytochemical constituents are indicated in Table 1.

Table 1- Results showing the Screening of Phytochemical constituents in the leaf extract of E. prostrata

\begin{tabular}{|l|l|l|}
\hline $\begin{array}{l}\text { S. } \\
\text { No }\end{array}$ & Plant Constituents & Presence or Absence \\
\hline 1. & Alkaloids & - \\
\hline 2. & Carbohydrates & + \\
\hline 3. & Glycosides & + \\
\hline 4. & Phytosterol & + \\
\hline 5. & Saponins & + \\
\hline 6. & Tannins and Phenolic compounds & + \\
\hline 7. & Protein and aminoacids & + \\
\hline 8. & Fixed oils and fats & - \\
\hline 9. & Flavanoid & + \\
\hline 10. & Lignin & + \\
\hline
\end{tabular}

Thin layer chromatographic plates spotted with ethanolic extract of leaves of E.prostrata. The band at Rf value 0.58 was identified as Wedelolactone. The identification of wedelolactone was done by the HPLC analysis Graph 1. The component separated out at a rentention time of 6.48 was identified as wedelolactone. The serum collected were subjected for different analysis and the results of SGOT, SGPT, ALP, bilirubin, , glutathione peroxidase, catalase, creatin ine, and glucose level in the serum. The results are provided in Table 2.

Table 2: Bioche mical analysis of 4-6 weeks male Wister alb ino rats

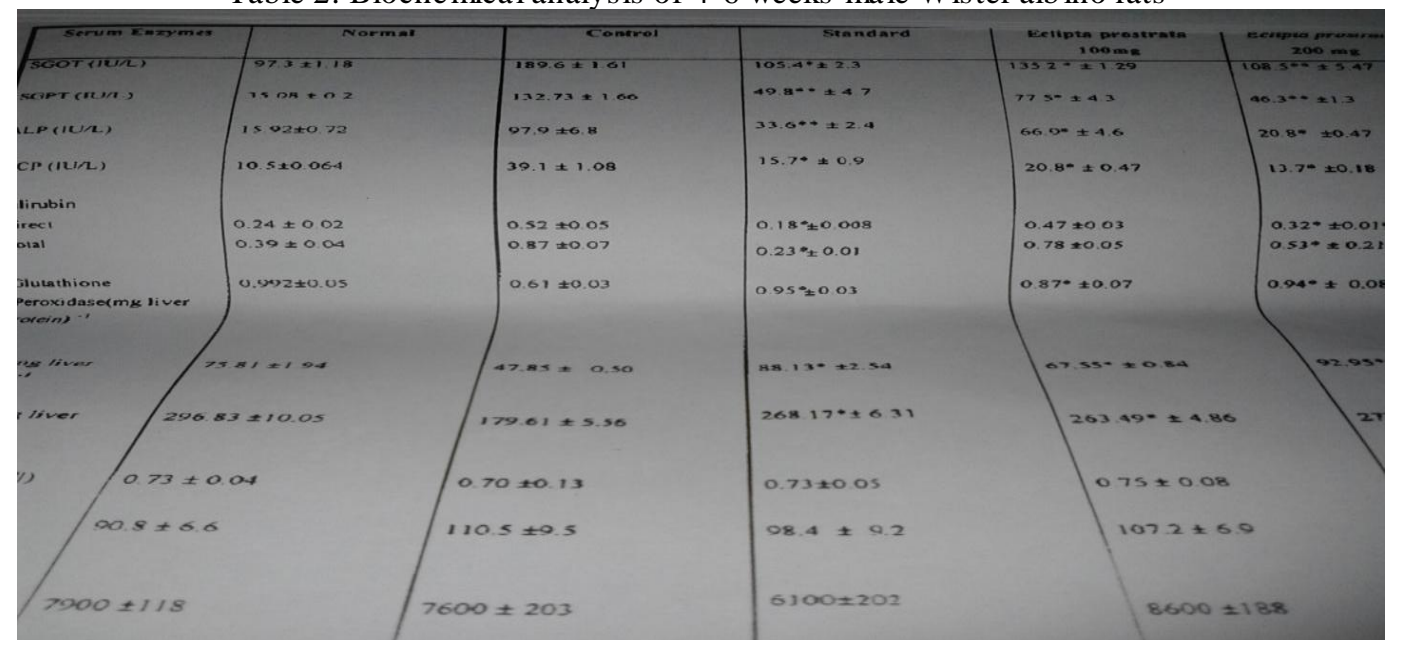


Histopathological results: An accurate estimator of the amount of liver cell inflammation or death can only be made by liver biopsy (Figure 2). The liver tissues are excised from normal, standard and E.prostrata treated male Wistar rates. The severity of liver disease due to $\mathrm{CCl} 4$ induction was compared with the normal tis sue of liver. The tissue from the animals treated with $200 \mathrm{mg} / \mathrm{KG}$ of the ethanolic extract of E. prostrate was found to be similar to that of animals treated with Silimarin. In the same manner, $100 \mathrm{mg} / \mathrm{Kg}$ of ethanolic extract of E. prostrate showed a positive protective effect, but lesser than that of Silimarin.

Graph 1: Identification of Wedelolactone by the HPLC analys is

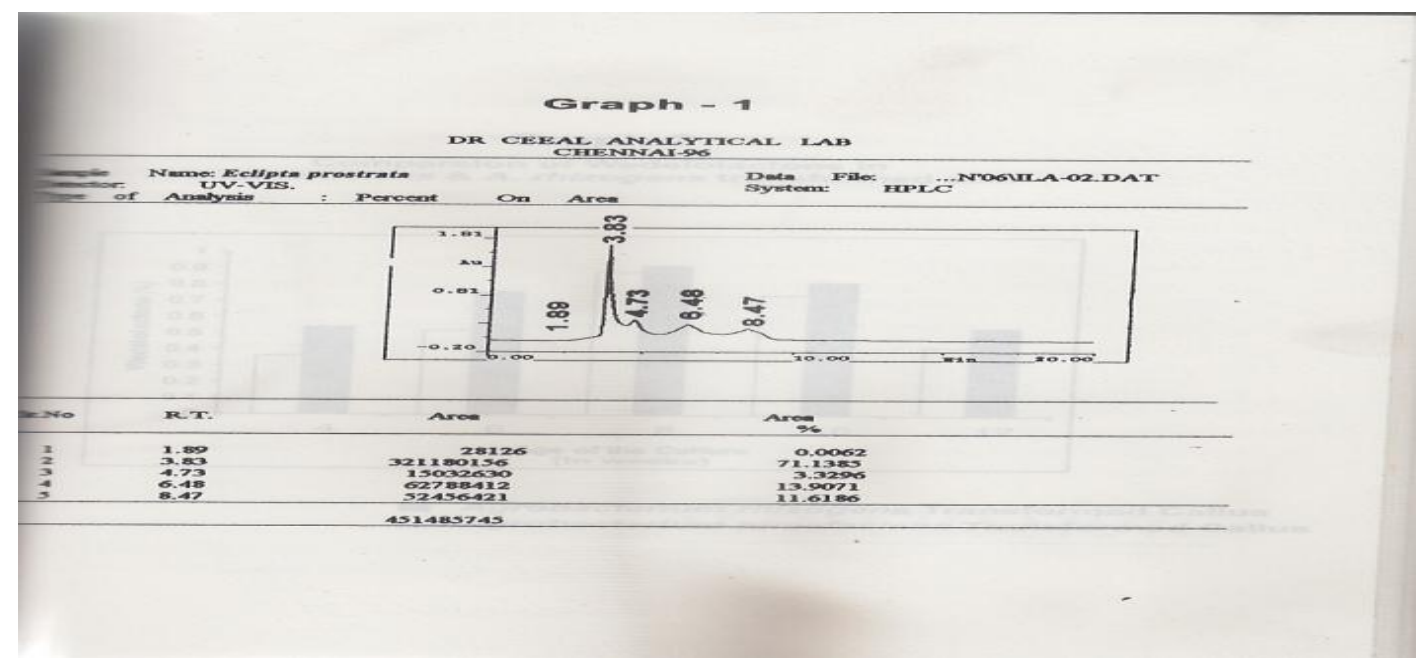

The presence of Wedelolactone is identified by thin layer chromatographyic method and RF value is found to be 5.6 instead of 5.8 observed by Das et a 1 (1990). About HPLC, the retention time of Wedelactone of our present study is 6.48 minutes, instead of 7 minutes reported in earlier studies. Primary liver diseas es (necrosis, obstructive jaundice, and carcinoma, viral or toxic hepatitis) as well as liver damage to the other causes result in elevated SGOT levels. The normal, standard values of liver function test of SGOT id 5-40 IU/L. As the SGOT levels of our present study is within the range. It denotes that $E$. prostrata is found to be effective in controlling the necrosis caused due to the CCL4 induced liver damages. Elevated level of SGPT is as sociated with liver disease or damage, myocardial infarction, muscularl dystrophy and cholecystitis. The normal value of liver function test of SGPT is 5-35 IU/L. The result of our present study is found to be within the range of the normal standard values, that indicates the efficacy of the drug against liver damages. Increase in the serum alkaline phos phatase level can lead to the elevated bile duct obstruction. It also causes the bone disorders in the elevated level. The normal value of alkaline phos phatase is $30-85 \mathrm{IU} / \mathrm{L}$.

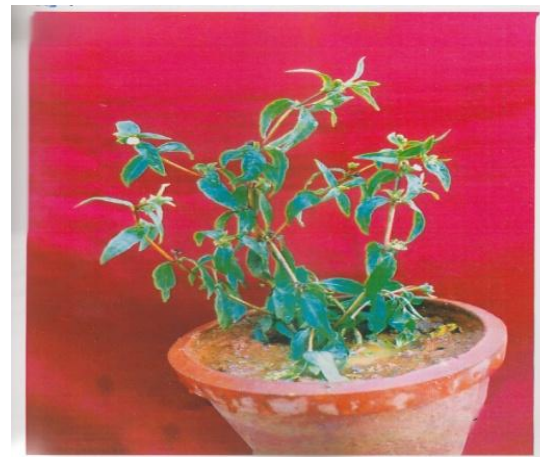

Fig 1 E. prostrate plant

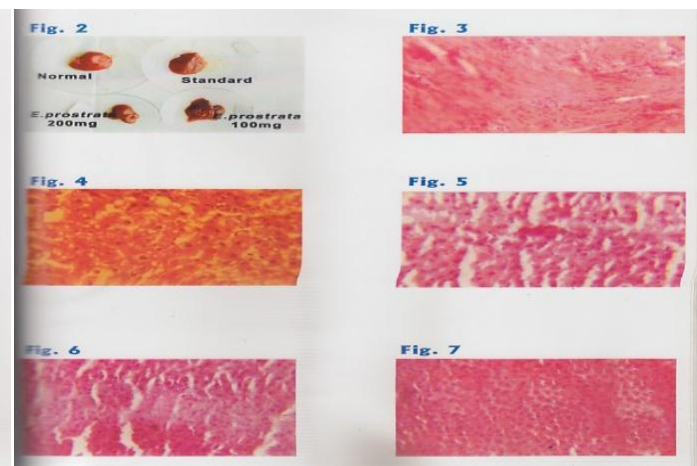

Fig 2 : Histopathological studies

In our study, the range falls with in the normal values. Hence the efficacy against E. prostrata is proved. Large amount of acid phosphatase is present in adult prostrate tissues in prostatic secretion. The normal value of ACP is 5-27 U/L. As the result of our present study is within the standard range infers the positive efficacy against prostratic secretion. Increased levels of bilirubin are associated with biliary obstruction and hepatocellular diseases as well as hemolytic disorders in adults and newborns. The normal value of direct bilirubin is $0.0-0.4 \mathrm{mg} / \mathrm{dL}$. As the result obtained in our study is within the range, it denotes that E. prostrate controls the biliary obstruction. The glutothine peroxidase, SOD, catalase, creatinine and glucose is found to be more or less similar to the normal values. Dosage levels of E.prostrata administered to the rats, both found to 
contain the hepatoprotective action against CCL4 induction. It was found that the dose of $100 \mathrm{mg} / \mathrm{Kg}$ of body weight. Hence the present study of E. prostrata, experimentally proved that it is found to be effective drug in the treatment of hepatic damages. Histopathological studies demonstrated the carbon tetrachloride causes fecal necrosis, portal infiltration, fatty change, kupffer cell hyperplasia and hydropic change. In the treated groups, necrosis, which is more severe form of injury, is markedly prevented; milder form of injury like fatty change and reduced necrosis persisted in extracts. CCL4 is metabolized by the mixed function of oxidase system in the endoplasmic reticulum of the liver. Cleavage of the carbon-chloride bond results in the formation of free trichloro methyl radicals (CCL3) which are highly unstable and immediately react with me mbrane components.

They form covalent bonds woth unsaturated fatty acids or abstract a hydrogen atom from the unsaturated fatty acids of membrane lipids, resulting in the production of chloroform and lipid radicals which react with molecular oxygen, this initiates peroxidative decomposition of phospholipids in the endoplasmic reticulum. The peroxidation process results in the release of soluble products that affect other membranes, such as cell membranes. It has been found that microsomal oxidation of chloroform results in the formation of phosgene. It is thought that a secondary metabolite causes cell death. Thus protective agents, Wedelolactone and demethyl wedelolactone of leaves of E. prostrata against CCL4 mediated lipid peroxidation by inhibiting the generation of free radical derivatives or as a result of the antioxidant activity of the protective agent itself.

\section{Conclusion}

Standardization of E. prostata $L$. was done by various physiochemical analyses and is also comparative to the stantdards as stated in the Indian Pharmacopoeia. Screening of ethanolic extacts of $E$. prostata revealed the presence of various phytochemicals. Identification of the active principle, Wedelolactone was done by various chromatographic methods such as HPLC and TLC. Therapeutic efficacy of E. prostrate was evaluated using male Wistar rats and it was found that the curative effect of E. prostata was comparable to that of that standard drug Silimarin. The optimum effective dosage was found to be $200 \mathrm{mg}$ of ethanolic extract per body weight of the experimental animals used. The plant E. prostata. L. under the present study is found to be more significant in clinical use ad depicted in the pharmacological studies. The drug was standardized pharmacognostically and the optimum dosage was found to be $200 \mathrm{mg} / \mathrm{kg}$ body weight of the animal. Technically and economically feasible protocol was developed for optimization of the secondary metabolite of E. prostrate. The plant E. prostata. L. under the present study is found to be more significant in clinical use ad depicted in the pharmacological studies. The drug was standardized pharmacognostically and the optimum dosage was found to be $200 \mathrm{mg} / \mathrm{kg}$ body weight of the animal. Technically and economically feasible protocol was developed for optimization of the secondary metabolite of E. prostrata.

\section{References}

[1]. Rajiv Sinha, K., Shewta Sinha, 2001. Ethnobiology- SurabhaiPublications, Rasta sanghiji, S.M.S. Highway, Jaipur, PP:1 -3

[2]. Wink M., 1988. Plant breeding: Importance of secondary metabolites for protection against pathogens and herbivores - Theoretical and Applied.Genet., 75: 225-233.

[3]. Sivarajan V. V. and Balachandran L., 1994, Ayurvedica Drugs and their plant sources; Oxford \& IBH Publishing co. pvt.Ltd, New Delhi, 91.

[4]. Singh P., and Bhargava S., 1992 Phytochemistry, 31, 2883.

[5]. Wagner H., and Fessler B., 1986, Planta Med, 5, 374

[6]. Kapoor. L.D., 1990, Handbook of Ayurvedic MedicinalPlants; CRC Press, Florida, 169.

[7]. Sikoria, B.C., Srivastava, S.J. and Niranjan, G.S., 1982. Phytochemical studies on Eclipta alba. J. Inc.chem.Soc., 59(7); 905.

[8]. Mroginshi K., Vora, K.K. and Seth, K.K., 1981. Effect of Picrorhiza Kurroa and Eclipta alba on Na+K+ ATPase in hepatic injury by hepatotoxic agemts. Indian J. Pharm., 13:253-259

[9]. Thiyagarajan, S.P., Thiruneelakandan, K., Subramanian, S. and Sundaravadivelu, T., 1982. Invitro inactivation of HBs. Ag. By Eclipta alba Hassk and Phyllanthas niruri L., Ind J. med. Res., 76: 124-130.

[10]. Sankaran, J.R., 1980. Tefroli in the management $\mathrm{f}$ viral hepat it is. (A single blind clinical study). Antiseptic, $77: 643$-646

[11]. Sing, V.K.., George, C.X., Gupta, B.M. 1983. Antiviral activity of plant extract Liv. 52 in mice experimentally infected with semlikli forest Encephalitis virus. Sci. Cul., 49: 354-355.

[12]. Chandra, T., Saidque, J. and Somasundaram, S., 1987. Effect of Ecliptaalba on inflammation and liver injury. Fittoter., 58 (1): 2332.

[13]. Govindachari, T. R., Nagarajan, K. and Pai, B. R., 1956a. Chemical examination of Wedelia calandulacea. Part 1, Structure of wedelolact one. J. Chem. Soc., 15B: 629-632.

[14]. Govidacheri, T. R., Nagarajan, K, and pai, B. R., 1956b. Wedelolactone from Eclipta alba. J. Sci. Ind. Res., 15B:664-665.

[15]. Bhargava, K.K., Krishnaswamy, N.R. and Seshadri, T. R., 1972. Desmethyl wedelolactone glucoside from Eclipta alba leaves. Ind. J. Chem., 8: 810-811.

[16]. Das, N., Bhavsar, G.C., Chauhan, M.G., 1990. Spectrophotometric determination of hepatoprotective principles of Eclipta alba. Ind. Drugs, 28(8): 356-358.

[17]. Ladue, J.S., Wroblewski F., and Karmen A., 1954, Serum glutamate oxaloacetate transaminase in human acute transmural mycocardial infarction, Science, 120, 497-499. 